\title{
Improving Traffic State Prediction Model for Variable Speed Limit Control by Introducing Stochastic Supply and Demand
}

\author{
Yuwei Bie $\mathbb{D}^{1},{ }^{1}$ Mudasser Seraj, ${ }^{1}$ Can Zhang, ${ }^{1}$ and Tony Z. Qiu $\mathbb{D}^{1,2}$ \\ ${ }^{1}$ Department of Civil and Environmental Engineering, University of Alberta, Edmonton, Canada \\ ${ }^{2}$ Intelligent Transportation Systems Research Center, Wuhan University of Technology, Wuhan, China \\ Correspondence should be addressed to Tony Z. Qiu; zhijunqiu@ualberta.ca
}

Received 19 March 2018; Revised 6 July 2018; Accepted 24 July 2018; Published 5 August 2018

Academic Editor: David Z. W. Wang

Copyright (c) 2018 Yuwei Bie et al. This is an open access article distributed under the Creative Commons Attribution License, which permits unrestricted use, distribution, and reproduction in any medium, provided the original work is properly cited.

\begin{abstract}
Variable speed limit (VSL) is becoming recognized as an effective way to improve traffic throughput and road safety. In particular, methods based on traffic state prediction exhibit promising potential to prevent future traffic congestion and collisions. However, field observations indicate that the traffic state prediction model results in nonnegligible error that impacts the next step decision making of VSL. Thus, this paper investigates how to eliminate this prediction error within a VSL environment. In this study, the traffic state prediction model is a second-order traffic flow model named METANET, while the VSL control is model predictive control (MPC) based, and the VSL decision is discrete optimized choice. A simplified version of the switching mode stochastic cell transmission model (SCTM) is integrated with the METANET model to eliminate the prediction error. The performance of the proposed method is assessed using field data from a VSL pilot test in Edmonton, Canada, and is compared with the prediction results of the baseline METANET model during the road test. The results show that during the most congested period the proposed SCTM-METANET model significantly improves the prediction accuracy of regular METANET model.
\end{abstract}

\section{Introduction}

The VSL control method was designed to keep the credibility of speed limits even under adverse conditions such as congestion and bottleneck road segments, so that the speed limits still work to maintain traffic safety and the highest possible traffic throughput. This method adjusts the posted speed limit based on real-time road, traffic, and weather conditions [1]. The VSL control method performs well in terms of control scale, control method flexibility, and feasibility. The most commonly used VSL strategies take a reactive approach with simple policies: using this strategy, the VSL decision is triggered by abnormal traffic states detected in real time, such as high traffic flow or low average speed. However, to achieve improved traffic throughput, a more complex control algorithm that includes optimized VSL decision-making is needed. Proactive VSL control goes one step further than reactive VSL control since it is optimized and calculated based on the predicted traffic state, so as to prevent a severe traffic breakdown from happening. Between two general patterns of VSL control, stimulation-reactive pattern and proactive pattern, this paper considers the latter pattern to be more challenging but promising.

In proactive VSL control, an accurate macroscopic level traffic prediction model is the core part. Over the last decades, various traffic prediction models were invented: the author classifies them to statistical and nonstatistical methods [2]. The statistical methods can predict the traffic flow with high accuracy under stable traffic conditions, commonly used methods including the class of Time Series models, such as seasonal Autoregressive Integrated Moving Average model [3] and Kalman Filter State-Space model [4], Neural Network [5], and Nonparametric Regression [6]. However, in the case of VSL control, traffic flow prediction is required under congestion condition at bottleneck road sections where traffic conditions can change abruptly, so that the author chose nonstatistical methods, i.e., the traffic prediction model derived from traffic flow models. Those models have potential to reflect the nature of traffic flow and capture the fluctuation of traffic flow: the traffic flow is treated as fluid that is aggregated 
by individual vehicles, and three variables are capable of describing the characteristics of traffic stream; they are flow, density, and mean speed. The traffic flow models are classified as first-, second-, or higher-order models, depending on the number of the included differential equations [7]. First-order model is able to describe traffic flow, but not traffic density or speed. Among all first-order models, the most used is Lighthill-Whitham-Richards model $[8,9]$ using one partial differential equation to describe the vehicle flow conservation law. This was also the first time that traffic flow model is combined with static fundamental diagram. Another representative first-order model is the Cell Transmission Model $[10-12]$, which is a discretized and simplified version of the Lighthill-Whitham-Richards model. The Payne model [13] is the oldest second-order traffic flow model. Besides the flow conservation law equation, the Payne model also includes one partial differential equation that describes mean speed dynamics. This model can replicate traffic phenomenon with higher accuracy. There are also other types of second-order traffic prediction models and combinations, such as variation kinematic waves [14], second-order traffic flow model with Kalman filter [15], and CTM-based second-order traffic flow model with particle filtering [16].

To achieve the highest traffic prediction accuracy, in this paper, a second-order traffic flow model named METANET $[17,18]$ is chosen. It is a discretized and enhanced version of the Lighthill-Whitham-Richards model combined with the Payne model. As it is a second-order traffic flow model, it is able to predict traffic density, average vehicle speed, and traffic flow by three dynamic functions in the model. METANET model fulfills the simplicity and convenience requirement since it has a space-time discrete, explicit analytical statespace form and allow for convenient discretization intervals [7]. What is more, METANET model is proved by previous studies that it is of high accuracy and relatively easy to calibrate, which makes it one of the most frequently utilized macroscopic traffic flow models in variety of traffic engineering tasks and researchers, and this model is capable of being used for optimal real-time traffic control of freeway traffic.

However, due to the stochastic nature of traffic flow, accurately predicting the traffic state of bottlenecks for VSL control is a complex task, especially during peak hours and for congested road segments. The traffic flow, speed, and density are by nature stochastic and variable, when traffic flow models are used for predicting traffic condition for making VSL control decisions, those stochasticity and variability need to be described and modeled and be added to the original static model to meet the specific high prediction requirement of VSL control. VSL field experiments in this paper have demonstrated that the traffic state prediction model exhibits larger error during the afternoon peak period. The stochastic nature of traffic can be modeled by adding an exogenous disturbance term to each round of calculation. This paper is inspired by the stochastic elements in the stochastic cell transmission model (SCTM) [19] and incorporates them in this study. The most straightforward method to model the stochasticity is to apply a sequential Monte Carlo simulation method to the CTM to mimic the change from free flow traffic conditions to congested conditions [20], but this method has a high computation cost. Another way to incorporate the stochastic element is to set the sending and receiving flow as random variables [21]. However, this method does not function well when traffic is light or congested, for the random terms cannot represent transportation features. In 2011, Sumalee et al. [19] proposed a stochastic CTM (SCTM) to describe the macroscopic dynamics of traffic flow under demand and supply uncertainties. Its switching mode design simplifies the sequential Monte Carlo method, and its stochastic element takes into account the fundamental diagram parameters instead of purely random generated. The SCTM has been implemented in practical research projects, such as dynamic journey time estimation [22] and road network traffic assignment under adverse weather [23]. The cell transmission model (CTM) is a first-order traffic flow model with no exogenous input, and it serves as the density dynamic function in the METANET model. In this paper, the regular density dynamic function in METANET model, also called CTM is replaced with the SCTM. Stochastic elements are added in anticipation of better prediction performance.

Besides traffic prediction model, the VSL control structure also has various choices [24] such as case-based reasoning, knowledge-based systems (in which the policy is based on previous practices), and model-based prediction. To find the optimal control structure that utilizes the traffic state prediction model, a MPC framework [25] is applied in this paper, forming a closed loop. The future traffic state is predicted, and then the optimal VSL decision is made based on prediction results. MPC is an optimal control scheme applied within a rolling horizon framework. Optimal control was successfully applied by Kotsialos et al. [26] to coordinate or integrate traffic control measures. Both optimal control and MPC have the advantage of a controller that generates an optimal control signal or decision according to a user-defined objective function. The objective function in this paper is a weighted summation of total travel time (TTT) and total travel distance (TTD), where the total travel time is minimized and total travel distance is maximized simultaneously.

VSL control has been successfully implemented in European countries and the United States [27]. In Edmonton, Canada, a four-week VSL field test was implemented on a 10-kilometer stretch of freeway, and this field test serves as a case study in this paper. The field data truly reflect the driver behavior and compliance of people in a major western Canadian city. The actual field data and METANETpredicted traffic data are extracted for comparison with the simulated data generated by the proposed model. This paper first integrates the SCTM to the METANET model for VSL control purposes and then tests its feasibility using both field experiment data and computer simulation data. The upgrading of METANET model, the field test result analysis, and the simulation provide references for future local researches.

The following sections of this paper include a literature review of VSL and the original SCTM model; an explanation 
of the model predictive control- (MPC-) based VSL algorithm; a description of the modified SCTM that is used in this study; presentation of the case study; and, finally, a discussion of the results and conclusion.

\section{A Model Predictive Control Based Variable Speed Limit Algorithm}

The model description is separated into four parts: the description of traffic state prediction model and the description of fundamental diagram used in this study. Section 2.1 will be notations and assumptions. Section 2.2 will describe in detail the baseline VSL control logic and algorithm. Section 2.3 will present model calibration skills including the calibration of fundamental diagram and the macroscopic traffic prediction model involved in VSL control.

2.1. Notations and Assumptions. For consistency, the freeway is divided into $N$ sections with lengths $\Delta_{i}, i=1, \ldots, N$, each having at most one on-ramp and off-ramp. All the variables used throughout this paper are defined as follows:

\section{$T$ : data collection interval $=20 \mathrm{~s}$ \\ $\lambda_{i}$ : number of lanes at section $i$ \\ $\Delta_{i}:$ length of section $i$}

$q_{i}(k)$ : number of vehicles in the freeway section $i$ at time $k \cdot T$ divided by the length $\Delta_{i}$

$\rho_{i}(k)$ : traffic density at time step $k$, section $i$.

$v_{i}(k)$ : average vehicle travel speed at time step $k$, section $i$.

$u_{i}(k)$ : the optimized speed limit posted at time step $k$, section $i$.

$r_{i}(k)$ : on-ramp flow at time step $k$, section $i$

$s_{i}(k)$ : off-ramp flow at time step $k$, section $i$

$V\left(\rho_{i}(k)\right)$ : desire speed in speed dynamics in METANET model

$\Theta$ : set of unknown parameters of METANT model

$\rho_{c r, i}$ : critical density of FD at section $i$

$\rho_{\text {jam }, i}:$ jam density of FD at section $i$

$v_{f, i}$ : free flow speed of FD at section $i$

$w_{i}$ : shockwave speed of FD at section $i$

$\theta$ : capacity drop fraction of $\mathrm{FD}$ at section $i$

TTT: total travel time

TTD: total travel distance

$\alpha_{\text {TTT }}$ : weight of TTT

$\alpha_{\text {TTD }}$ : weight of TTD.

2.2. The MPC-Based VSL Algorithm Using METANET Prediction Model. The MPC-based VSL algorithm is composed of five parts: an original traffic prediction model, control case traffic prediction model, optimizer, objective function, and constraints. As shown in Figure 1, for VSL control road

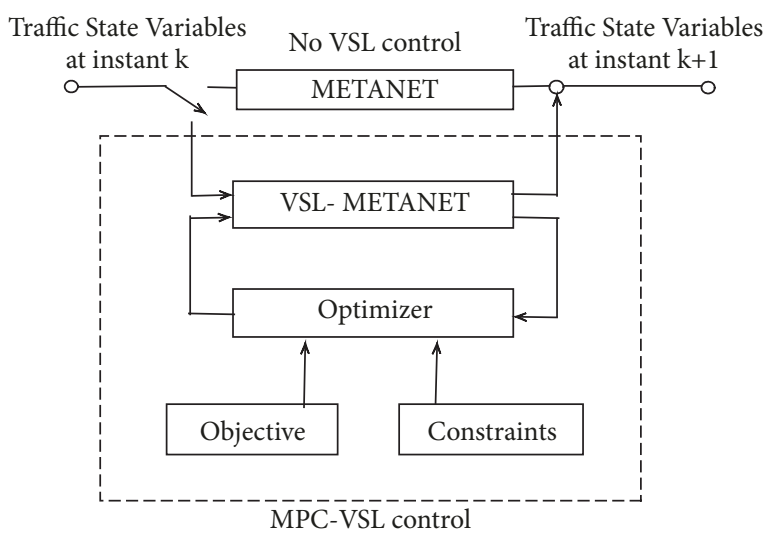

FIGURE 1: The block diagram of model predictive VSL control.

segments, the system uses a modified METANET model for traffic state prediction, and the discrete choice optimizer makes a choice based on predicted traffic states, given the objective function and constraints. After optimization, the optimal VSL choice is shown to operators, and, simultaneously, the VSL value is inputted into the VSL-METANET model again for the final traffic state prediction results to be recorded. Figure 1 shows a block diagram of the VSL control system.

The METANET model as a second-order traffic flow model introduces speed dynamic functions instead of including only density dynamics to describe flow conservation law. This feature of the METANET model enables it to predict density, speed, and flow variables, respectively, accurately, as well as with a small time interval. The discretized model formulation of the original METANET is written as follows.

Density dynamics (also referred to the regular CTM) are as follows:

$$
\begin{aligned}
\rho_{i}(k+1) & \\
= & \rho_{i}(k) \\
& +\frac{T}{\Delta_{i} \lambda_{i}}\left[\lambda_{i-1} q_{i-1}(k)-\lambda_{i} q_{i}(k)+r_{i}(k)-s_{i}(k)\right]
\end{aligned}
$$

Speed dynamics are as follows:

$$
\begin{aligned}
v_{i}(k+1)= & v_{i}(k)+\frac{T}{\tau}\left[V\left(\rho_{i}(k)\right)-v_{i}(k)\right] \\
& +\frac{T}{\Delta_{i}} v_{i}(k)\left[v_{i-1}(k)-v_{i}(k)\right] \\
& -\frac{\eta \cdot T\left[\rho_{i-1}(k)-\rho_{i}(k)\right]}{\tau \cdot \Delta_{i} \cdot\left[\rho_{i}(k)+\kappa\right]}
\end{aligned}
$$

Flow dynamics are as follows:

$$
q_{i}(k+1)=\rho_{i}(k+1) \cdot v_{i}(k+1)
$$

where $i$ is the index of links, and $i=1,2, \ldots M$, with $M$ representing the number of testbed sections. $K$ is the index of time instants, and $T$ is the calculating time interval, where 


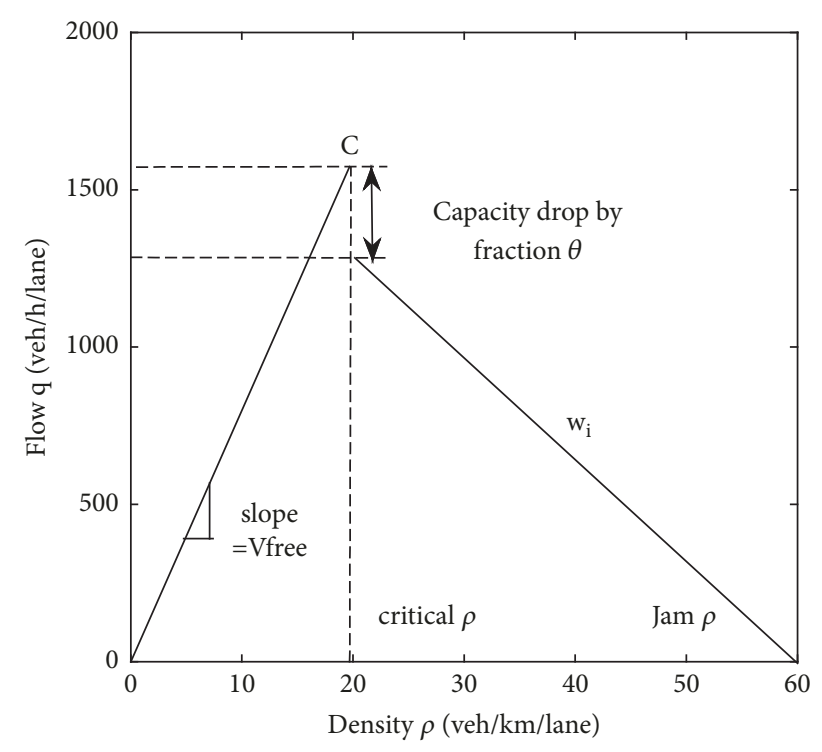

FIGURE 2: The triangular FD assumption.

$T=20$ s. $\Delta_{i}$ represents the segment length of link $i$, and $\lambda$ is the lane number of link $i$. In the model, $\tau, \kappa, \alpha$, and $\eta\left(\mathrm{km}^{2} / \mathrm{h}\right)$ are global model parameters calibrated using the historical data. The desired speed $V\left(\rho_{i}(k)\right)(\mathrm{km} / \mathrm{h})$ in the speed dynamics is represented by the following expression:

$$
V\left(\rho_{i}(k)\right)=v_{f, i} \exp \left[-\frac{1}{\alpha}\left(\frac{\rho_{i}(k)}{\rho_{c, i}}\right)^{\alpha}\right]
$$

The triangular fundamental diagram (FD) is assumed in the control algorithm. Figure 2 shows the triangular FD assumption used in this paper. In (4), $v_{f, i}$ is free flow speed and $\rho_{c, i}$ represents critical density, which is the density associated with capacity. In this model, the density dynamics do not involve parameters, and other parameters in the speed dynamics are calibrated globally for goodness of fit. Of the four terms that make up the speed dynamics equation, each term has a physical meaning. In (2), the second term is referred to as the relaxation term, indicating that, with a lag time item $\tau$, the mean speed $v$ of the link "relaxes" to the desired speed, which largely depends on the parameters of the FD. The selection of the desired speed is critical in reflecting driver behavior, and, based on previous practice [28], the format of (4) was chosen. The third term is the convection term used to indicate that vehicles traveling from the upstream link $i-1$ to the current link $i$ adapt their speed gradually rather than instantaneously. The fourth term is the anticipation term, indicating that drivers are always watching the road ahead for changes in traffic. If a driver observes high traffic density in the downstream link $i+1$, the driver then reacts by slowing down. The constant $\kappa>0$ is added to keep the anticipation term limited when density is low.

The METANET algorithm for the VSL control environment is modified by replacing the desired speed with a modified one. In the control segments, the desired speed is assumed to be the posted advisory speed, rather than the speed determined by the fundamental diagram as in the noncontrol case. In the VSL-METANET model, the desired speed term then becomes the optimized VSL value, as decided by the optimizer in the MPC system.

Desired speed term when VSL is triggered is as follows:

$$
V\left(\rho_{i}(k)\right)=u_{i}(k)
$$

The objective function minimizes a weighted sum of TTT and TTD in a discrete choice fashion. In terms of definition, TTT indicates a weighted sum of density of all time steps and links. Only minimizing TTT produces system bias, wherein the optimizer tends to choose a lower speed limit to decrease the density, so that TTD is maximized simultaneously. In the objective function, weights $\alpha_{T T T}$ and $\alpha_{T T D}$ are applied to TTT and TTD.

$$
\begin{aligned}
& T T T=T \sum_{j=1}^{p r-1} \sum_{i=1}^{M} \lambda_{i} L_{i} \rho_{i}(k+j) \\
& T T D=T \sum_{j=1}^{p r-1} \sum_{i=1}^{M} \lambda_{i} L_{i} \rho_{i}(k+j) v_{i}(k+j) \\
& J=T \sum_{j=1}^{p r-1} \sum_{i=1}^{M} \lambda_{i} L_{i}\left[\alpha_{T T T} \rho_{i}(k+j)\right. \\
& \left.-\alpha_{T T D} \rho_{i}(k+j) v_{i}(k+j)\right]
\end{aligned}
$$

The desired speed under the VSL environment is decided by the optimizer. The optimization is subjected to several constraints of traffic safety, driver acceptance, and traffic flow characteristics [1]. The first constraint is the upper limit of $u_{i}(k)$, since the regular speed limit is $80 \mathrm{~km} / \mathrm{h}$, under congested conditions $u_{i}(k) \leq 80$. The second constraint is to maintain continuous traffic flow even under congestion. From local regulations, the lower bound of the speed limit is $30 \mathrm{~km} / \mathrm{h}$, which means $u_{i}(k) \geq 30$. In Canada, the speed limit is in multiples of $10 \mathrm{~km} / \mathrm{h}$; therefore, to ensure safety, the change in speed limit between two time instants is 0 or $10 \mathrm{~km} / \mathrm{h}$, represented as $u_{i}(k)-u_{i}(k+1) \in\{-10,0,10\}$. The optimizer then becomes a discrete choice model.

2.3. Calibrating the FD and METANET Model. The parameter estimation of the FD is based on data collected by conventional loop detectors. For the parameter calibration of the $\mathrm{FD}$, free flow speed $\mathrm{v}_{f, i}$, critical density $\rho_{c r, i}$, capacity $C_{i}$, jam density $\rho_{\text {jam }, i}$, and capacity drop fraction $\theta$ must be estimated. For calibration, the data format used is $\left(\rho_{i}, q_{i}\right)$ data points, where the horizontal coordinate is density and the vertical coordinate is flow, as Figure 2 shows. The procedure for obtaining the necessary parameters from a triangular FD is as follows.

(1) The identification of capacity $C_{i}$ : in defining the triangular $\mathrm{FD}$, the summit point of the triangle roughly indicates critical density and capacity. All $\left(\rho_{i}, q_{i}\right)$ points are plotted to find the third largest $q_{i}$ and then take it as capacity $C_{i}$. The largest flow is not chosen because it is normally an extreme outlier; this could be due to detection error so that the third largest flow is empirically chosen as capacity. 
(2) The identification of critical density $\rho_{c r, i}$ : after determining $C_{i}$, the corresponding traffic density $\rho_{i}$ is then identified as critical density $\rho_{c r, i}$. This identification is based on the assumption of triangular shape FD: the vertical line across $C_{i}$ intersects the horizontal density axis at critical density $\rho_{c r, i}$. Point $\left(\rho_{c r, i}, C_{i}\right)$ is then the vertex of the triangular FD.

(3) The identification of free flow speed $v_{f, i}$ : after defining $\rho_{c r, i}$, the whole dataset can be divided into two parts, the left and right side of the triangle. The left side of the triangle represents uncongested traffic conditions and the right side of the triangle represents congested traffic conditions. Then the slope of each data point distributed on the left side is calculated, and the average is taken as $v_{f, i}$, as shown in the following equation [2]:

$$
v_{f, i}=\frac{1}{n}\left(\sum_{i i=1}^{n} \frac{q_{i i}}{\rho_{i i}}\right), \quad \rho_{i i} \in\left(0, \rho_{c r, i}\right)
$$

(4) The identification of jam density $\rho_{\text {jam, },}$, the capacity drop fraction $\theta$, and shockwave speed $w_{i}$ : the jam density $\rho_{\text {jam }, i}$ represents a theoretical value that indicates when the road section is totally congested and all vehicles have stopped moving. From the dataset, the data point with the largest density value and zero speed is selected as $\rho_{\text {jam }, i}$. The slope of the right side, i.e., shockwave speed $w_{i}$ is determined by least square fit fixing one foot $\left(\rho_{\text {jam }, i}, 0\right)$. After determining one foot and the slope of the right side, the intersecting point of the regression line and the vertical line across $C_{i}$ is the congested capacity after a capacity drop fraction of $\theta$.

For the parameter estimation of METANET model, $\tau$, $\kappa, \alpha$, and $\eta\left(\mathrm{km}^{2} / \mathrm{h}\right)$ are global model parameters to be calibrated. The calibration of parameters is not done in real time, according to the logic that both parameters from FD and METANET model represent aggregated traffic behavior of a period of time, the calibration happens right before the field test and uses traffic data collected in the last 7 days. In doing SCTM-METANET simulation, the same parameter set as in the field test is used. The parameters of METANET model are estimated via the following expression:

$$
\begin{aligned}
\Theta^{*} & =\arg \min _{\Theta}\left[\sum_{i i}^{N}\left\{v^{i i}{ }_{o b s}-f\left(v^{i i}{ }_{o b s} \mid \Theta\right)\right\}^{2}\right. \\
& \left.+\sum_{i i}^{N}\left\{\rho^{i i}{ }_{o b s}-f\left(\rho^{i i}{ }_{o b s} \mid \Theta\right)\right\}^{2}\right]
\end{aligned}
$$

\section{Integrating Switching-Mode SCTM with METANET Model}

The switching-mode SCTM is employed to replace the regular CTM in the METANET model. The traditional CTM, derived from the conservation law of traffic flow, is a single linear system without exogenous input or tuning parameters. When CTM is used for traffic state prediction, after rounds of iteration, the model is farther and farther from reality since during the calculation time the outside traffic condition changes quickly. In this paper, the traffic prediction length

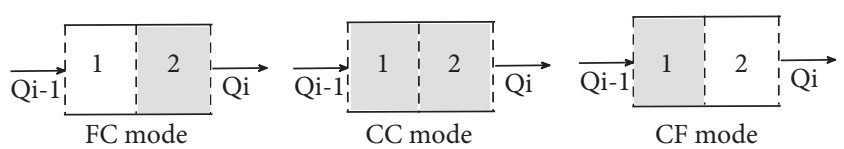

Figure 3: Traffic operation modes.

in the VSL field test is 5 minutes, containing 15 iterations of calculation, but congestion can form within 3 minutes. To solve this problem, the SCTM introduces exogenous stochastic disturbance terms to each iteration of computing to counteract the prediction error.

Incorporating stochastic supply and demand into a traffic flow model can be different ways, the FD stochasticity, the boundary condition stochasticity, and stochastic adjusting parameter if necessary. In the case of the SCTM in this paper, the stochastic elements are added in two ways: (1) demand stochasticity, the inflow disturbance term added to the CTM; (2) supply stochasticity also FD stochasticity, each of the disturbance terms containing stochastic FD parameters extracted by statistical technics.

Another feature of the SCTM is that, under different traffic condition modes, the disturbance elements change slightly. The original SCTM has five modes (refer to [19]), and in this paper the number of modes is simplified to three because the CTM is still used for free flow conditions and the wave front of congestion is only from downstream to upstream. The three modes are free flow to congestion (FC) mode, congestion to congestion (CC) mode, and congestion to free flow (CF) mode. Different from the CTM, the SCTM considers the adjacent two cells as a whole and uses vector $P(k)=\left[\rho_{1}(k), \rho_{2}(k)\right]^{T}$ in the density dynamics calculation. Please see Figure 3 for the illustration of traffic operation modes where white segment represents for low traffic density free flow condition and grey segment represents for high traffic density congested condition.

When defining the traffic operational modes, a typical SCTM develops complicated probabilistic occurrence models as mode switches [19], and it serves generalized traffic situations. However, when SCTM is implemented in a specific VSL control road segment whose traffic condition is highly repeatable and well understood, the probability expression then decays to a fixed version. The fixed mode switch time points are empirically set based on traffic data, which can be observed in the case study of this paper. Additionally, the switch time considers the suggestion of local authorities. Free flow mode, which is the original density dynamic function in METANET, is in effect 4:00pm to 4:59pm. The FC mode takes effect during 5:00pm to 5:09pm and serves as a transitional stage between free flow mode and congested mode. The CC mode takes place between $5: 10 \mathrm{pm}$ and $5: 49 \mathrm{pm}$. Then the CF mode takes effect between 5:50pm and 5:59pm. From 6:00pm on, the CTM takes over again. In the FC mode, $u(k)=\left(q_{i-1}(k), r_{i}(k), s_{i}(k), q_{i}(k)\right)^{T}, \omega_{2}(k)=w_{c, 2}(k)$, and $\lambda(k)=\left(0, \rho_{\text {jam }, 2}(k)\right)^{T}$.

Density dynamics (SCTM) under FC mode are

$$
\begin{aligned}
P(k+1)= & \left(I+A_{2} \omega_{2}(k)\right) P(k)+B_{2} \omega_{2}(k) \lambda(k) \\
& +B u(k)
\end{aligned}
$$


where

$$
\begin{aligned}
A_{2} & =\left[\begin{array}{cc}
0 & \frac{T_{s}}{l_{1}} \\
0 & -\frac{T_{s}}{l_{2}}
\end{array}\right], \\
B_{2} & =\left[\begin{array}{cc}
0 & -\frac{T_{s}}{l_{1}} \\
0 & \frac{T_{s}}{l_{2}}
\end{array}\right], \\
B & =\left[\begin{array}{lll}
\frac{T_{s}}{l_{1}} & -\frac{T_{s}}{l_{1}} & 0 \\
0 & 0-\frac{T_{s}}{l_{2}} & \frac{T_{s}}{l_{2}}
\end{array}\right] .
\end{aligned}
$$

After the traffic condition transitions to congestion mode, the CC model applies. In the CC model, $\omega_{i}(k)=w_{i}(k)$, and $\lambda(k)=\left(\rho_{\text {jam }, 1}(k), \rho_{\text {jam }, 2}(k)\right)^{T}$.

Density dynamics (SCTM) under CC mode are

$$
\begin{aligned}
P(k+1)= & \left(I+\sum_{i=1}^{2} A_{i} \omega_{i}(k)\right) P(k) \\
& +\sum_{i=1}^{2} B_{i} \omega_{i}(k) \lambda(k)+B u(k)
\end{aligned}
$$

where

$$
\begin{aligned}
A_{1} & =\left[\begin{array}{rr}
-\frac{T_{s}}{l_{1}} & 0 \\
0 & 0
\end{array}\right], \\
A_{2} & =\left[\begin{array}{cc}
0 & \frac{T_{s}}{l_{1}} \\
0 & -\frac{T_{s}}{l_{2}}
\end{array}\right], \\
B & =\left[\begin{array}{llll}
0 & 0 & 0 & 0 \\
0 & 0 & -\frac{T_{s}}{l_{2}} & -\frac{T_{s}}{l_{2}}
\end{array}\right],
\end{aligned}
$$

After the traffic condition transitions out of congestion mode, the CF model applies. In the CF model, $\omega_{1}(k)=w_{1}(k)$, $\omega_{2}(k)=\mathrm{v}_{f, 2}(k)$, and $\lambda(k)=\left(\rho_{\text {jam }, 1}(k), \mathrm{C}_{1}(k)\right)^{T}$.

Density dynamics (SCTM) under CF mode are

$$
\begin{aligned}
P(k+1)= & \left(I+\sum_{i=1}^{2} A_{i} \omega_{i}(k)\right) P(k) \\
& +\left(B_{0}+\sum_{i=1}^{2} B_{i} \omega_{i}(k)\right) \lambda(k)+B u(k)
\end{aligned}
$$

where

$$
\begin{aligned}
& A_{1}=\left[\begin{array}{rr}
-\frac{T_{s}}{l_{1}} & 0 \\
0 & 0
\end{array}\right], \\
& A_{2}=\left[\begin{array}{ll}
0 & 0 \\
0 & -\frac{T_{s}}{l_{2}}
\end{array}\right], \\
& B_{0}=\left[\begin{array}{rr}
0 & -\frac{T_{s}}{l_{1}} \\
0 & \frac{T_{s}}{l_{2}}
\end{array}\right], \\
& B_{1}=-A_{1}, B=B_{2}=0 .
\end{aligned}
$$

\section{Case Study}

The vehicle detection stations (VDS) on a 10-kilometer corridor of Whitemud Drive in Edmonton, Canada, collect, and store traffic data from dual loop detectors. This section of road plays an important role in people and freight transportation in the city. The VDS system currently has 28 VDS in total, and each station has three or four dual loops. The data recording frequency is 20 seconds. Each dual loop reports the volume $q$-the number of vehicles crossing the loop detector during a 20-second time interval-and mean speed measurement $v$, and the occupancy measurement, which cannot be used directly in traffic control and cannot be transformed accurately into density. The accurate density measurement $\rho$ is calculated by $\rho=q / v$. Figure 4 shows the testbed geometry, VDS locations and the location of dynamic message signs (DMS) that will be used to display variable speed limits.

The VSL control decisions are made and sent out by software with the METANET model embedded. When the detected average speed is below the posted speed of $80 \mathrm{~km} / \mathrm{h}$, the VSL-METANET prediction is triggered on the corresponding road segment. The prediction step length is 20 seconds and 15 iterations, which means the prediction horizon is 5 minutes. The prediction is calculated every minute. So that all variables for comparison are $1 \mathrm{~min}$ aggregated, and when simulating SCTM-METANET the same aggregation level is used. Theoretically speaking, the METANET model can be made to predict farther future such as $10 \mathrm{~min}$ and $15 \mathrm{~min}$; however, in real life situation in Edmonton, Canada especially during peak hours, traffic condition changes drastically even within 5 minutes (please see Figures 6(b), 6(c), and 6(d) and Figures 7(b), 7(c), and 7(d)). So that when doing municipal level experiment on freeways, prediction horizon longer than 5 minutes was rarely used. If under non-peak hours when traffic flow is relatively steady, it is possible to recalibrate METANET model and achieve high accuracy prediction for longer horizons.

During real-time VSL control, data from loop detector stations are sent to computers in the traffic management center in the City of Edmonton. The software installed in the computers is responsible for providing speed limit 


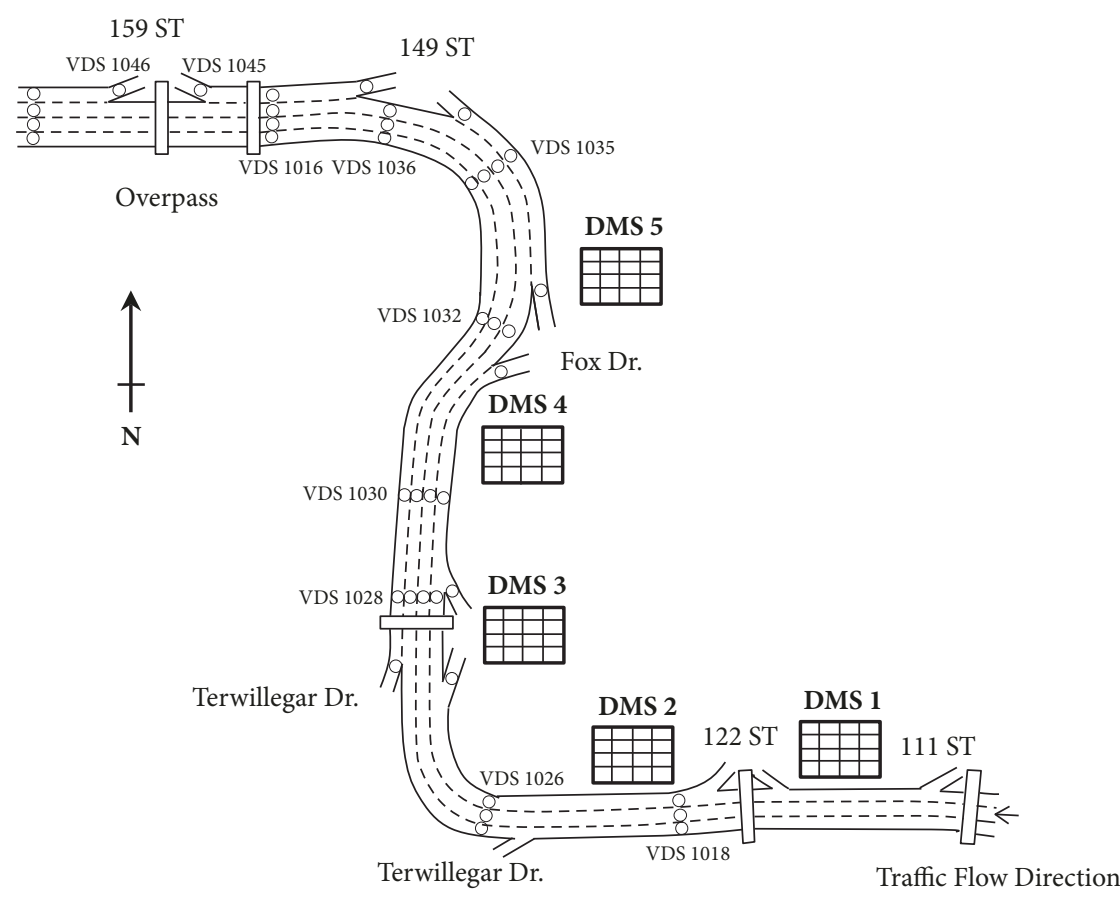

$\circ$ Loop Detector

LED Dynamic Message Sign (DMS)

Figure 4: Vehicle detection stations and dynamic message signs on Whitemud Drive, Edmonton, Canada.

suggestions. The decisions are then shown on screen with beeping notifications for human operators to check and send to DMS on the road. From August 13 to September 4 of 2015 on weekdays, the City of Edmonton conducted a four-week VSL pilot test on westbound Whitemud Drive. Five DMSs in total were located upstream of predefined congestion-prone points. From the testing period, DMS 1 on August 17 was chosen as a case for this study because a period of normal peak hour congestion occurred and the VSL functioned well. Due to legal reasons, the VSLs were not mandatory to drivers, and they were labeled as "Advisory Driving Speed" on the DMSs.

The performance measurements of VSL in this study are TTT and TTD, which can be taken together as the measure of effectiveness (MOE), as shown in (6) and (7). At each time step, the TTT and TTD are calculated from a weighted sum of the density and flow of all links, in which the weight is the segment length of each link. Figure 5 shows the speed profile of the test day. VSL is observed to help stabilize the speed and the predefined fixed time FC-CC-CF mode switch makes sense in this case.

In the field test, the global parameters of METANET model were calibrated based on historical traffic data of August 2014, and, during field test, those parameters were fixed. The reason for fixing global parameter is to reduce the complexity of online traffic prediction. Table 1 shows the precalibrated global parameters of METANET model.

4.1. The Measurement of Prediction Error and the Performances of SCTM-METANET. The discrepancies between the actual

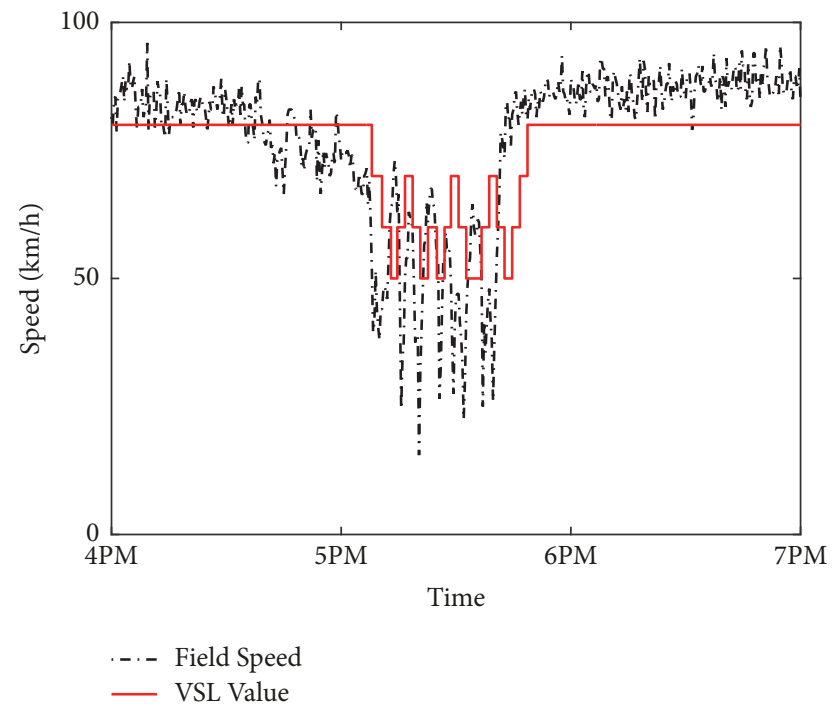

FIGURE 5: Speed profile of case study day Aug.17, 2015.

MOE and those predicted can be categorized as structured error and unstructured error. Structured error occurs when fixed parameters, such as segment lengths, are incorrectly measured. Unstructured error is the true error or the error that is found in the prediction model, which usually happens after eliminating structured error.

The structured error (SE) of the predicted MOE is assumed to be proportional to the original predicted MOE, after denying other assumptions such as proportion to 
TABle 1: Parameters used in the field test.

\begin{tabular}{|c|c|c|c|c|}
\hline MET N NET Parometerc & $\eta$ & $\kappa$ & $\alpha$ & $\tau$ \\
\hline MELANET Parameters & 37.98 & 10.00 & 2.29 & 120.00 \\
\hline FD Parameters & $\begin{array}{c}w_{1}, w_{2} \\
12\end{array}$ & $\begin{array}{c}\mathrm{v}_{f, 1}, \mathrm{v}_{f, 2} \\
82\end{array}$ & $\begin{array}{c}\rho_{\text {jam }, 1}, \rho_{\text {jam }, 2} \\
118\end{array}$ & \\
\hline
\end{tabular}

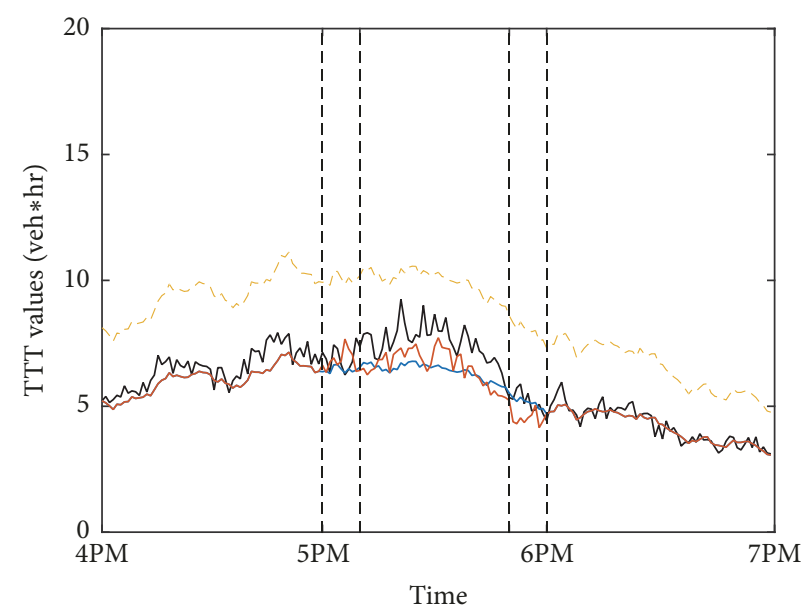

(a)

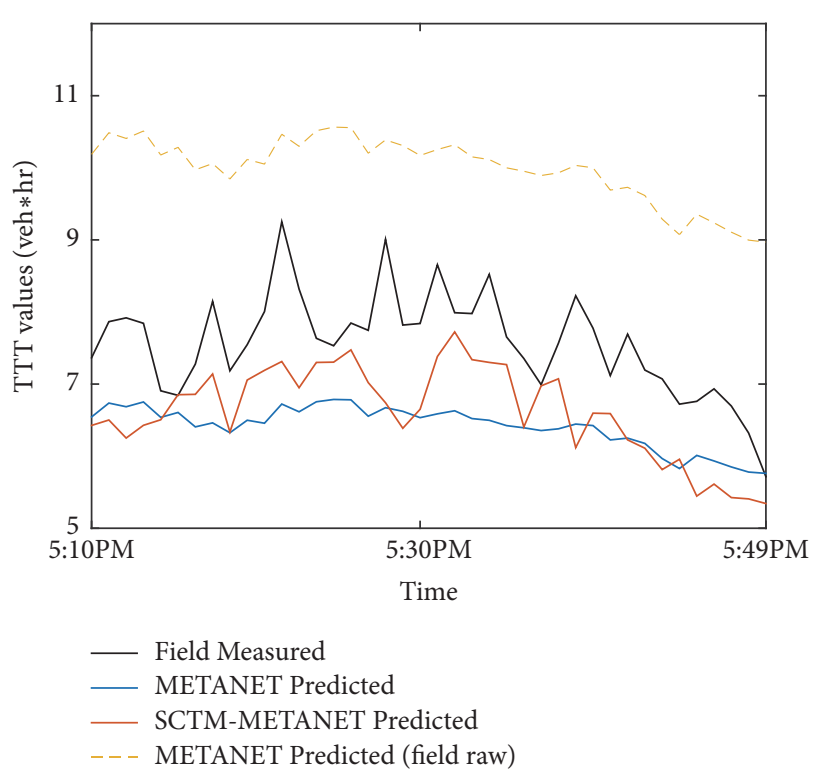

(c)

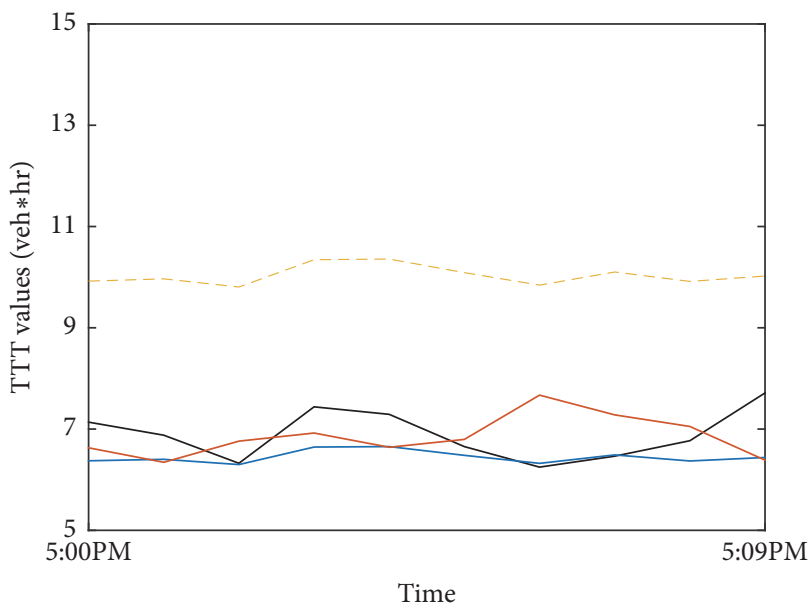

Field Measured METANET Predicted

- SCTM-METANET Predicted METANET Predicted (field raw)

(b)

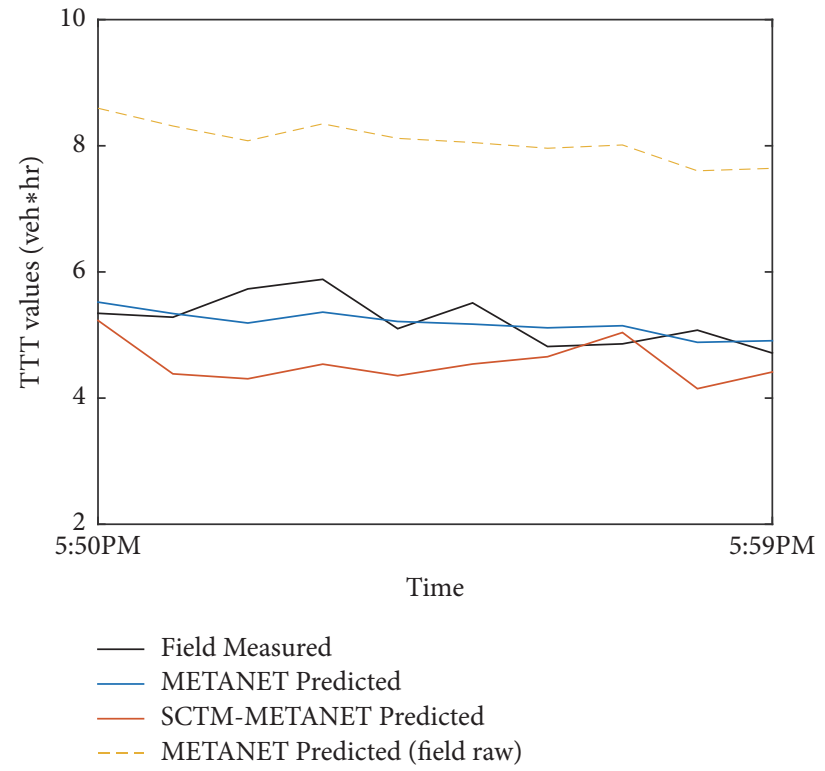

(d)

Figure 6: (a) Comparing field-measured TTT, original METANET, and SCTM-METANET predicted TTT; (b), (c), (d) detailed view of FC, $\mathrm{CC}$, and CF modes.

measured MOE, fixed value of error, and normal distribution. So that the adjusted predicted MOE is calculated by scaling down original predicted MOE. The reference point is the beginning of the study period 4:00:00PM, meaning the suggestion that at the start point of this time period no structured prediction uncertainty existed. Based on the above understanding, the overall structured uncertainty can be calculated. In Figures 6 and 7, SE refers to the distance between the curve METANET-predicted (field raw) and the curve METANET-predicted. 


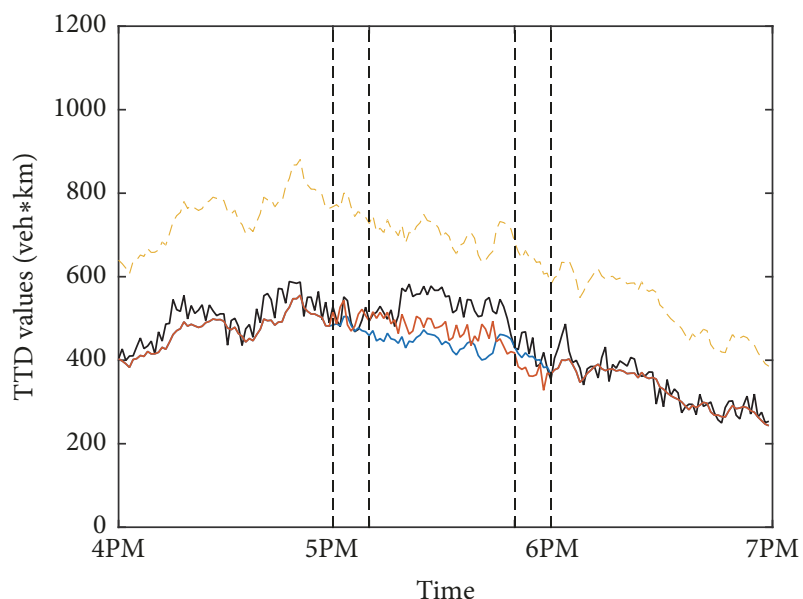

(a)

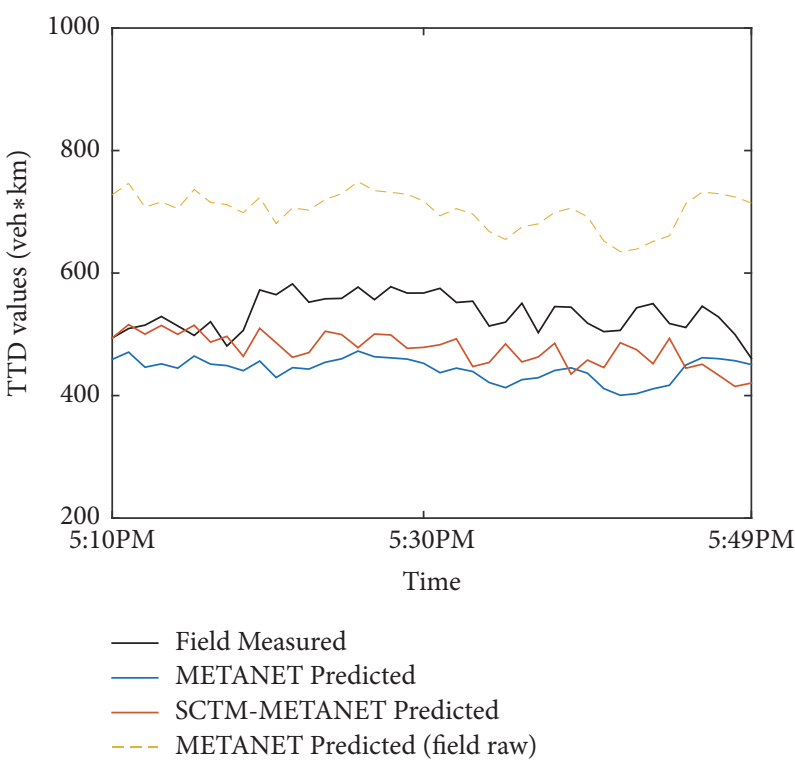

(c)

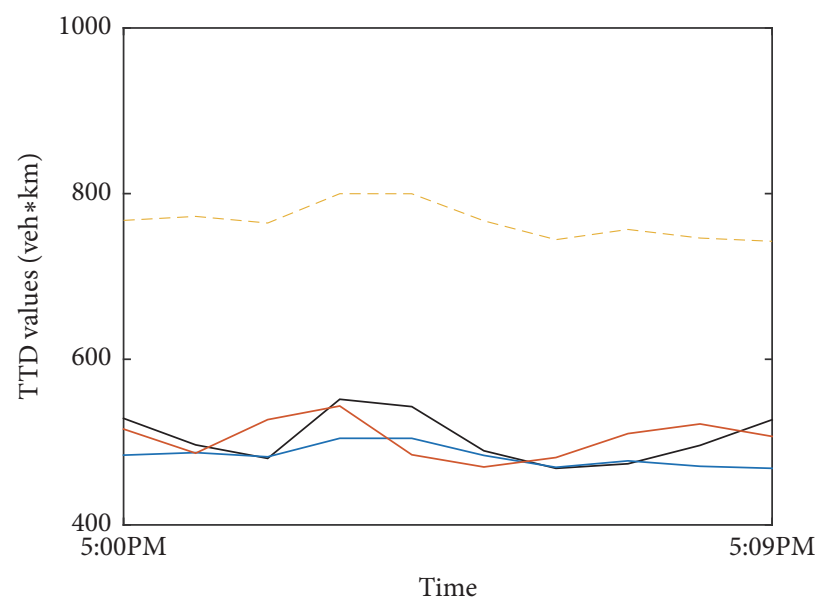

— Field Measured

— METANET Predicted

- SCTM-METANET Predicted

- - - METANET Predicted (field raw)

(b)

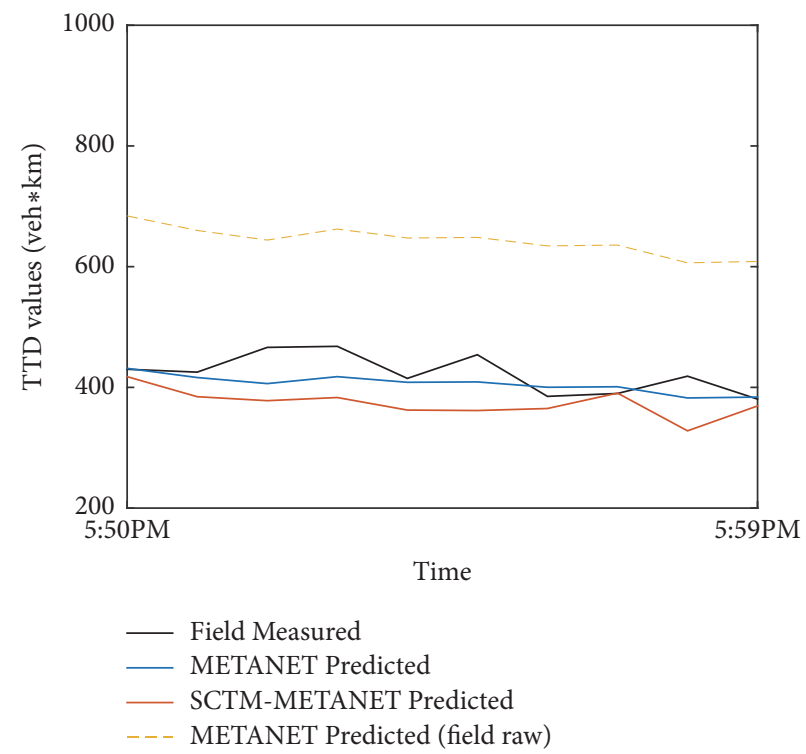

(d)

FIGURE 7: (a): Comparing field-measured TTD, original METANET, and SCTM-METANET predicted TTD. (b), (c), (d) detailed view of FC, CC, and CF modes.

$$
S E(t)=\left(1-\left.\frac{\operatorname{Moe}_{m}\left(t^{\prime}\right)}{\operatorname{Moe}_{p}\left(t^{\prime}\right)}\right|_{t^{\prime}=4: 00: 00 P M}\right) \cdot \operatorname{Moe}_{p}(t)
$$

where $\mathrm{Moe}_{m}(t)$ and $\mathrm{Moe}_{p}(t)$ represent field-measured and METANET-predicted TTT and TTD, respectively, at time step $t$. After eliminating structured error by scaling down the original predicted MOE, unstructured error (UE) remains, which represents the true performance of the prediction model. In Figures 6 and 7, unstructured error (UE) refers to the difference between the curve METANETpredicted and field-measured. In the following section, the root mean square error (RMSE) of both the original and new models is calculated from the UE.

$$
\begin{aligned}
U E(t)= & \operatorname{Moe}_{m}(t)-\operatorname{Moe}_{p}(t) \\
& \left.\cdot \frac{\operatorname{Moe}_{m}\left(t^{\prime}\right)}{\operatorname{Moe}_{p}\left(t^{\prime}\right)}\right|_{t^{\prime}=4: 00: 00 P M}
\end{aligned}
$$

Figures 6 and 7 and Tables 2 and 3 demonstrate the performance of the proposed SCTM-METANET versus the regular METANET. The raw field data predicted by METANET are collected from the field control software, and, after eliminating SE, the data become METANET-predicted data, 
TABLE 2: Quantitative comparison of METANET and SCTM-METANET models: TTT.

\begin{tabular}{lccccc}
\hline RMSE of TTT prediction & $\begin{array}{c}\text { Free Flow } \\
(4: 00-4: 59 \mathrm{pm})\end{array}$ & $\begin{array}{c}\text { FC Mode } \\
(5: 00-5: 09 \mathrm{pm})\end{array}$ & $\begin{array}{c}\text { CC Mode } \\
(5: 10-5: 49 \mathrm{pm})\end{array}$ & $\begin{array}{c}\text { CF Mode } \\
(5: 50-5: 59 \mathrm{pm})\end{array}$ & $\begin{array}{c}\text { Free Flow } \\
(6: 00-6: 59 \mathrm{pm})\end{array}$ \\
\hline Conventional METANET & 0.40 & 0.61 & 1.27 & 0.31 & 0.19 \\
SCTM-METANET & -- & 0.77 & 1.09 & 19.84 & -- \\
Improvement (Percentage) & 0 & $-26 \%$ & 40 & $171 \%$ & 0 \\
No. of Data Points & 60 & 10 & & & 60 \\
\hline
\end{tabular}

TABLE 3: Quantitative comparison of METANET and SCTM-METANET Models: TTD.

\begin{tabular}{lccccc}
\hline RMSE of TTD prediction & $\begin{array}{c}\text { Free Flow } \\
(4: 00-4: 59 \mathrm{pm})\end{array}$ & $\begin{array}{c}\text { FC Period } \\
(5: 00-5: 09 \mathrm{pm})\end{array}$ & $\begin{array}{c}\text { CC Period } \\
(5: 10-5: 49 \mathrm{pm})\end{array}$ & $\begin{array}{c}\text { CF Period } \\
(5: 50-5: 59 \mathrm{pm})\end{array}$ & $\begin{array}{c}\text { Free Flow } \\
(6: 00-6: 59 \mathrm{pm})\end{array}$ \\
\hline Conventional METANET & 20.0 & 31.42 & 95.97 & 31.53 & 25.5 \\
SCTM-METANET & -- & 29.76 & 67.96 & 60.69 & -- \\
Improvement (Percentage) & 0 & $5 \%$ & $29 \%$ & $42 \%$ & 0 \\
No. of Data Points & 60 & 10 & 40 & & 60 \\
\hline
\end{tabular}

which are ready to be compared with the computer-simulated SCTM-METANET.

Figure 6 shows that during free flow before 5PM and after 6PM the regular METANET model satisfactorily captures the trend of traffic flow. During 5:10PM and 5:49PM, the most congested period, the original METANET largely underestimates the TTT as well as its fluctuation. After introducing the SCTM into METANET, the predicted TTT not only is closer to reality, but also better matches the oscillating trend of the field-detected TTT. Figures 6(a) and 6(c) show steady improvement of the proposed SCTM-METANET model. Under transitional mode FC and CF, the SCTM-METANET does not show visible improvement of prediction accuracy, which may be due to too few data points. The FC and CF mode last only for 10 minutes, which means each condition has only 30 data points for computation. In summary, the SCTM-METANET outperforms the regular METANET model with noticeable improvements in stability, especially during the most congested period. Those improvements can be observed not only in the figure, but also quantitatively in Table 2.

When focusing on the prediction error during the CC period, the proposed SCTM-METANET model shows a significant $14 \%$ improvement calculated from 40 data points. Quantitative comparison also shows that, during the FC mode and CF mode, the proposed model shows unstable performance with negative improvements. The free flow period before and after SCTM-METANET shows stable and best prediction accuracy with the lowest error.

In the prediction of TTD, the SCTM-METANET performs better than for TTT. Figure 7 shows that, during free flow hours before 5PM and after 6PM, the regular METANET model satisfactorily captured the traffic flow trend. Also, during 5:10PM and 5:49PM, the original METANET largely underestimated the TTD and its fluctuation. When using the SCTM-METANET, the predicted TTD was not only closer to reality, but also better matched the oscillating trend of the field-detected TTD. Figures $7(a)$ and $7(c)$ show steady improvement for proposed model. Under CF mode, the SCTM-METANET does not show improvement in prediction accuracy, as in the case of predicting TTT. Under FC, the proposed model shows overall improvement. The quantitative measurement of the prediction performance for TTD proves the above observation. During the CC period, the proposed model shows a significant reduction in error (29\%), which outperforms the TTT. Under the FC mode, the proposed model shows 5\% improvement compared to the baseline model, which is still more satisfying than the case of TTT. The details of quantitative performance comparison can be found in Table 3 .

\section{Conclusion and Remarks}

In this paper, a modified second-order traffic status prediction model, SCTM-METANET, is proposed for improving the performance of the regular METANET model during VSL control. In particular, the proposed model helped to counteract traffic statues prediction error during the most congested period. This study employed a switching mode SCTM model and integrated it with the METANET model so that under each of the FC, CC, and CF modes the SCTMMETANET had slightly different parameters. In addition, unlike the original METANET, the proposed model made predictions in a piecewise manner. The original METANET was already implemented in the VSL field test, and the predicted traffic variables were stored, so the collected field data were considered as ground truth data. The proposed SCTM-METANET was calibrated and simulated by a computer, using field data from the same period. Then the prediction results of the original METANET and SCTMMETANET models could be compared to the ground truth data. Data refining was applied to the field raw data of regular METANET predicted results. The concepts of SE and UE were introduced for clarity, and by eliminating SE the prediction result of regular METANET could then be compared to the SCTM-METANET model. The results clearly show that, 
during transitional modes FC and CF, the proposed model shows unstable performance, and, during the CC mode, the SCTM-METANET model largely reduces the prediction error of TTT and TTD by $14 \%$ and $29 \%$, respectively.

This paper also revealed that that both METANET and SCTM-METANET underestimate the traffic density especially within $5 \mathrm{pm} \sim 6 \mathrm{pm}$ when the traffic mode switches to congestion. The happening of this phenomenon is mainly due to the nature of loop detector system and the basic feature of CTM which is the core part of density prediction in both METANET and SCTM-METANET: the road is segmented as cells and it is assumed that within one cell the speed, density, and flow are all homogeneous, and the homogeneous condition transfers to the next cell. Based on this assumption, in the field the freeway is divided into cells which are approximately $500 \mathrm{~m}$ long and the loop detectors installed at the beginning of the cell collects and represents the traffic condition of the whole cell, the data collection interval is $20 \mathrm{~s}$. Under noncongested situation, the above hardware settings are abundant for accurately estimating traffic conditions. However, when during peak hour, traffic density, speed, and flow are not homogeneous in $500 \mathrm{~m}$ and many vehicles would enter the cell in the 20 s data collection interval. It is delightful to find that when upgrading METANET to SCTM-METANET, the underestimation is relaxed although not totally eliminated. That is because of the bringing in of supply and demand stochasticity: the model realizes that there exist more vehicles in the cell than previously estimated.

Future research may build on the results of this study by solving the model's problem of instability during the FC and CF modes. Since the current SCTM-METANET model was calibrated offline and with precalibrated parameters, future work with the SCTM-METANET model will involve obtaining parameters and conducting calibration in real time. In the future research, the utilizing of higher frequency data collection method and more location flexible data collection method such as connected vehicle will shed light upon this situation.

\section{Data Availability}

The data used in this research is collected by underpavement loop detectors on a segment of urban freeway called Whitemud Drive in Edmonton, Alberta, Canada. The loop detector data collected in the field is continuously sent to the traffic management center of City of Edmonton. The owner of the data is City of Edmonton, the local government. The data is shared by University of Alberta for research use. In this paper, the loop detector data as well as the posted advisory driving speed data was provided by City of Edmonton. To replicate the research results in this paper, one can ask the author for the raw data set but under the approval of City of Edmonton. And recently, all loop detector data of Edmonton can be visualized and downloaded from a public website, although the highest data frequency raw data cannot be downloaded. Using lower frequency loop detector data will partially reproduce the analysis result in this research. The website mentioned is http://www.its.ualberta.ca/wcpa/homeIndex/index.

\section{Disclosure}

The contents of this paper reflect the views of the authors who are responsible for the facts and the accuracy of the data presented herein. The contents do not necessarily reflect the official views or policies of the City of Edmonton and Transport Canada. This paper does not constitute a standard specification or regulation.

\section{Conflicts of Interest}

The authors declare that they have no conflicts of interest.

\section{Acknowledgments}

The authors would like to thank Wai Cheung, Janis Chow, Michael Vaudan, Adrian Loh, and Rahim Karmali from the traffic operation group in the City of Edmonton for providing the Whitemud Drive loop detector data. This research work was jointly supported by the Natural Sciences and Engineering Research Council (NSERC) of Canada, City of Edmonton, and Transport Canada.

\section{References}

[1] M. Hadiuzzaman, T. Z. Qiu, and X.-Y. Lu, "Variable speed limit control design for relieving congestion caused by active bottlenecks," Journal of Transportation Engineering, vol. 139, no. 4, pp. 358-370, 2013.

[2] Y. Bie, T. Z. Qiu, C. Zhang, and C. Zhang, "Introducing Weather Factor Modelling into Macro Traffic State Prediction," Journal of Advanced Transportation, vol. 2017, Article ID 4879170, 15 pages, 2017.

[3] B. M. Williams and L. A. Hoel, "Modeling and forecasting vehicular traffic flow as a seasonal ARIMA process: theoretical basis and empirical results," Journal of Transportation Engineering, vol. 129, no. 6, pp. 664-672, 2003.

[4] C. Antoniou, M. Ben-Akiva, and H. Koutsopoulos, "Online Calibration of Traffic Prediction Models," Transportation Research Record: Journal of the Transportation Research Board, pp. 235245, 2005.

[5] S. Afandizadeh and J. Kianfar, "A Hybrid Neuro-Genetic Approach to Short-Term Traffic Volume Prediction," International Journal of Civil Engineering, vol. 7, p. 41, 2009.

[6] B. L. Smith, B. M. Williams, and R. K. Oswald, "Comparison of parametric and nonparametric models for traffic flow forecasting," Transportation Research Part C: Emerging Technologies, vol. 10, no. 4, pp. 303-321, 2002.

[7] A. Spiliopoulou, M. Kontorinaki, M. Papageorgiou, and P. Kopelias, "Macroscopic traffic flow model validation at congested freeway off-ramp areas," Transportation Research Part C: Emerging Technologies, vol. 41, pp. 18-29, 2014.

[8] M. J. Lighthill and G. B. Whitham, "On kinematic waves. II. A theory of traffic flow on long crowded roads," Proceedings of the Royal Society A Mathematical, Physical and Engineering Sciences, vol. 229, pp. 317-345, 1955.

[9] P. I. Richards, "Shock waves on the highway," Operations Research, vol. 4, no. 1, pp. 42-51, 1956.

[10] C. F. Daganzo, "The cell transmission model: a dynamic representation of highway traffic consistent with the hydrodynamic 
theory," Transportation Research Part B: Methodological, vol. 28, no. 4, pp. 269-287, 1994.

[11] C. F. Daganzo, "A behavioral theory of multi-lane traffic flow. Part I: long homogeneous freeway sections," Transportation Research Part B: Methodological, vol. 36, no. 2, pp. 131-158, 2002.

[12] C. F. Daganzo, "A behavioral theory of multi-lane traffic flow. Part II: merges and the onset of congestion," Transportation Research Part B: Methodological, vol. 36, no. 2, pp. 159-169, 2002.

[13] H. J. Payne, "Models of freeway traffic and control," in Mathematical Models of Public Systems: Simulation Council Proceedings Series, pp. 51-61, 1971.

[14] J. A. Laval and C. F. Daganzo, "Lane-changing in traffic streams," Transportation Research Part B: Methodological, vol. 40, no. 3, pp. 251-264, 2006.

[15] Y. Wang and M. Papageorgiou, "Real-time freeway traffic state estimation based on extended Kalman filter: a general approach," Transportation Research Part B: Methodological, vol. 39, no. 2, pp. 141-167, 2005.

[16] L. Mihaylova, R. Boel, and A. Hegyi, "Freeway traffic estimation within particle filtering framework," Automatica, vol. 43, no. 2, pp. 290-300, 2007.

[17] M. Papageorgiou, J.-M. Blosseville, and H. Hadj-Salem, "Macroscopic modelling of traffic flow on the Boulevard Périphérique in Paris," Transportation Research Part B: Methodological, vol. 23, no. 1, pp. 29-47, 1989.

[18] M. Papageorgiou, H. Hadj-Salem, J. M. Blosseville, and N. Bhouri, Control, Computers, Communications in Transportation, Elsevier, 1990.

[19] A. Sumalee, R. X. Zhong, T. L. Pan, and W. Y. Szeto, "Stochastic cell transmission model (SCTM): a stochastic dynamic traffic model for traffic state surveillance and assignment," Transportation Research Part B: Methodological, vol. 45, no. 3, pp. 507-533, 2011.

[20] X. Sun, L. Munoz, and R. Horowitz, "Highway traffic state estimation using improved mixture Kalman filters for effective ramp metering control," in 42nd IEEE International Conference on Decision and Control (IEEE Cat. No. 0 3CH 37475), vol. 6, pp. 6333-6338, 2003.

[21] R. Boel and L. Mihaylova, "A compositional stochastic model for real time freeway traffic simulation," Transportation Research Part B: Methodological, vol. 40, no. 4, pp. 319-334, 2006.

[22] A. Sumalee, T. Pan, R. Zhong, N. Uno, and N. Indra-Payoong, "Dynamic stochastic journey time estimation and reliability analysis using stochastic cell transmission model: Algorithm and case studies," Transportation Research Part C: Emerging Technologies, vol. 35, pp. 263-285, 2013.

[23] A. Sumalee, K. Uchida, and W. H. K. Lam, "Stochastic multimodal transport network under demand uncertainties and adverse weather condition," Transportation Research Part C: Emerging Technologies, vol. 19, no. 2, pp. 338-350, 2011.

[24] A. Hegyi, B. De Schutter, and H. Hellendoorn, "Model predictive control for optimal coordination of ramp metering and variable speed limits," Transportation Research Part C: Emerging Technologies, vol. 13, no. 3, pp. 185-209, 2005.

[25] Y. Bie, Implementing Model Predictive Control based Variable Speed Limit on Urban Freeways: Data Imputation, Model Modification and Field Test Analysis, 2016.

[26] A. Kotsialos, M. Papageorgiou, and F. Middelham, "Optimal coordinated ramp metering with advanced motorway optimal control," Transportation Research Record, no. 1748, pp. 55-65, 2001.
[27] L. Elefteriadou, S. S. Washburn, Y. Yin, V. Modi, and C. Letter, Variable Speed Limit (VSL) - Best Management Practice, vol. 11, Florida Dep Transp Res Center, 2012.

[28] X. Wang, M. Seraj, Y. Bie, T. Z. Qiu, and L. Niu, "Implementation of variable speed limits: Preliminary test on Whitemud Drive, Edmonton, Canada," Journal of Transportation Engineering, vol. 142, no. 12, 2016. 


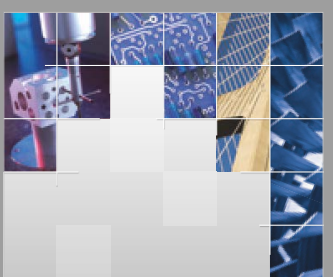

\section{Enfincering}
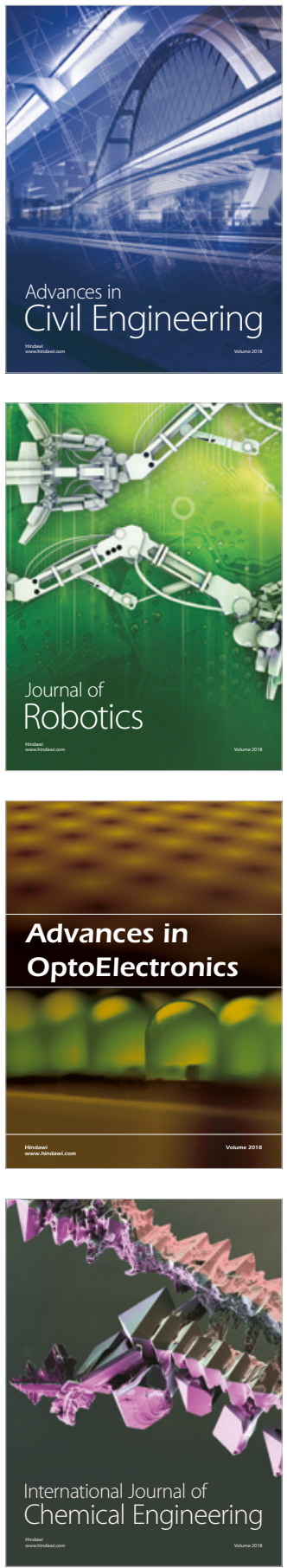

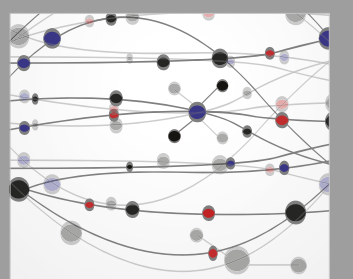

\section{Rotating \\ Machinery}

The Scientific World Journal

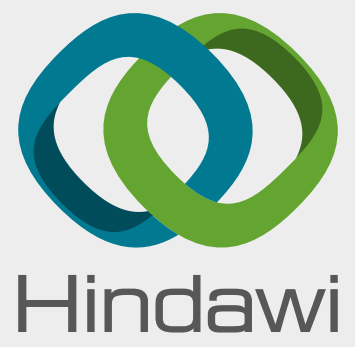

Submit your manuscripts at

www.hindawi.com
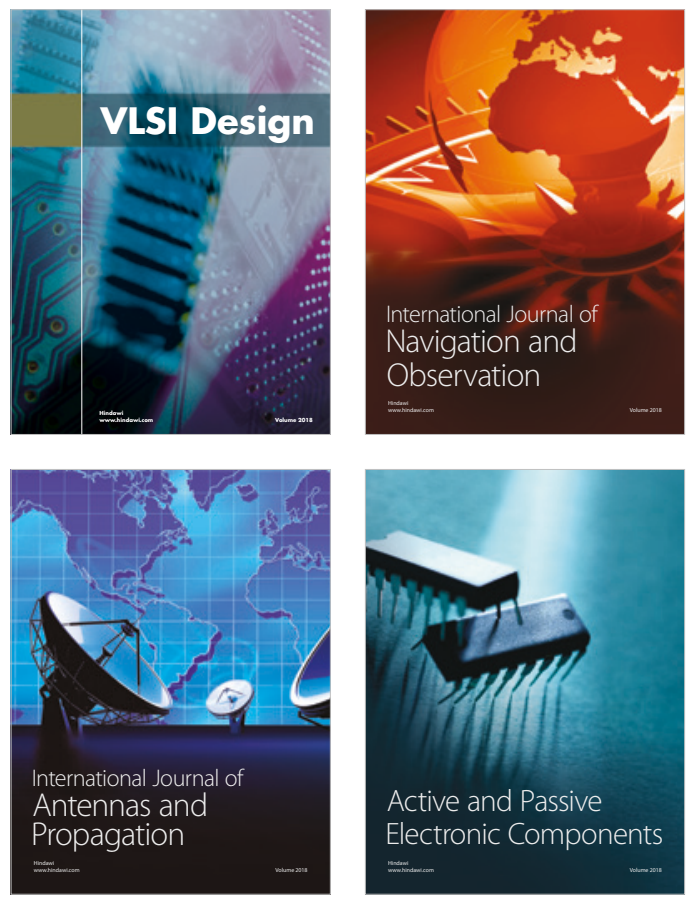
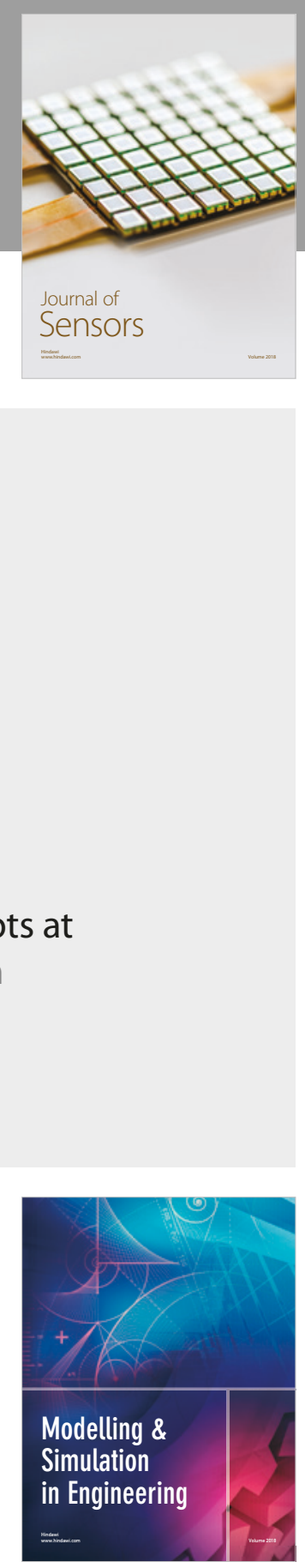

\section{Advances \\ Multimedia}
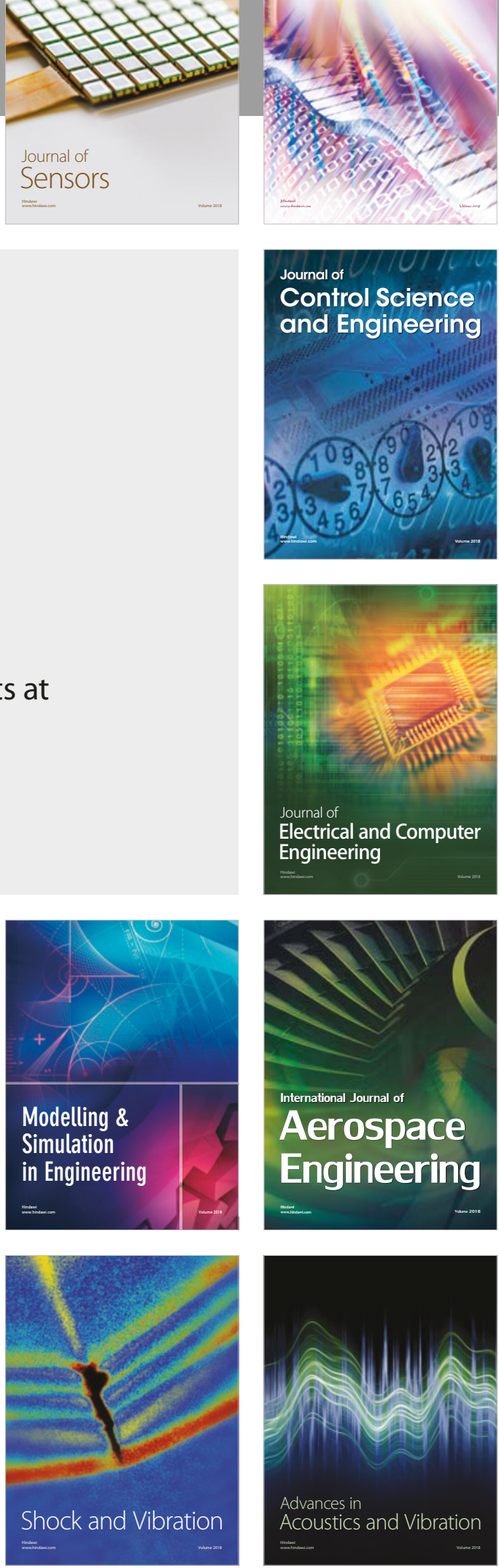